\title{
ESTUDO DA QUALIDADE DA GASOLINA TIPO A E SUA COMPOSIÇÃO QUÍMICA EMPREGANDO ANÁLISE DE COMPONENTES PRINCIPAIS
}

Fábio Israel M. Carvalho* e Heronides A. Dantas Filho

Faculdade de Química, Instituto de Ciências Exatas e Naturais, Universidade Federal do Pará, 66075-110 Belém - PA, Brasil

Recebido em 06/03/2013; aceito em 30/07/2013; publicado na web em 01/11/2013

\begin{abstract}
STUDY OF TYPE A GASOLINE QUALITY AND ITS CHEMICAL COMPOSITION USING PRINCIPAL COMPONENT ANALYSIS. The type A gasoline samples were analyzed by gas chromatography with flame ionization detector (GC-FID) which allowed quantifying and classifying of the various compounds into different classes of hydrocarbons. Several physicochemical parameters were evaluated according to the official methods in order to compare the results obtained against the limits established by the Agência Nacional de Petróleo, Gás Natural e Biocombustíveis (ANP, 2011). Additionally, principal component analysis (PCA) was applied to discriminate the samples studied, which revealed the separation of four groups according to their chemical composition determined in samples collected from the eight fuel distributors in the State of Pará.
\end{abstract}

Keywords: type A gasoline; GC-FID; PCA.

\section{INTRODUÇÃO}

A gasolina é um dos mais importantes produtos derivados do petróleo, obtida a partir dos processos de refino, sendo o segundo combustível mais consumido no Brasil, perdendo apenas para o óleo diesel. ${ }^{1}$ É um combustível líquido, volátil e inflamável, constituído por mais de 400 diferentes compostos, cuja composição final depende da origem do petróleo, dos processos de produção (destilação, alquilação, hidrocraqueamento, craqueamento catalítico, craqueamento térmico, reforma catalítica, entre outros). ${ }^{2,3}$ De acordo com a composição química, os hidrocarbonetos presentes na gasolina pertencem principalmente às classes das parafinas (normal e ramificado), olefinas, naftenos e aromáticos, e em menores quantidades, por compostos oxigenados; formados basicamente por moléculas com cadeias de 4 a 12 átomos de carbono, com pontos de ebulição variando entre 30 a $220{ }^{\circ} \mathrm{C} .{ }^{4,5}$ Além dos hidrocarbonetos, a gasolina também possui contaminantes tóxicos naturais em baixas concentrações, formados por compostos contendo enxofre, oxigênio, benzeno, metais e nitrogênio. ${ }^{5}$

No Brasil, a Agência Nacional de Petróleo, Gás Natural e Biocombustíveis (ANP) é o órgão responsável pela regulamentação e fiscalização da qualidade desse combustível, e especifica dois tipos de gasolinas, a tipo A, que é obtida diretamente nas refinarias, não sendo permitida sua venda em postos de combustíveis no Brasil, e a tipo $\mathrm{C}$, que é obtida a partir da mistura da gasolina tipo A com a adição de álcool etílico anidro combustível (atualmente $25 \pm 1 \%$ v/v), que é a comercializada no varejo. ${ }^{6}$

A ANP, através da Resolução no 57/2011, DOU de 21/10/2011 estabeleceu valores mínimos e máximos para o controle da qualidade dos combustíveis, em particular a gasolina, especificando ensaios físico-químicos para garantir a manutenção da qualidade da gasolina desde a produção até o consumo. Para isso, a ANP baseia-se em métodos padrões mediante o emprego de Normas Brasileiras (NBR) e Métodos Brasileiros (MB) estabelecidos pela Associação Brasileira de Normas Técnicas (ABNT) e pelas normas internacionais da American Society for Testing and Materials (ASTM). ${ }^{6}$

A aplicação de métodos rápidos e confiáveis de análise deste combustível tem sido favorecida com a utilização de padrões de referência que permitem a identificação e quantificação de componentes

*e-mail: fabioimc@yahoo.com.br desta matriz. Nos últimos anos, vários estudos tiveram como foco o desenvolvimento de novas metodologias analíticas para um maior controle da qualidade de combustíveis automotivos. ${ }^{7}$ Alguns destes trabalhos citam que as técnicas analíticas mais utilizadas para determinação da composição química de combustíveis são: a espectroscopia no infravermelho (IR), ${ }^{8-11}$ espectroscopia no ultravioleta (UV), ${ }^{12,13}$ espectrometria de massas (MS), ${ }^{7,14}$ ressonância magnética nuclear (NRM) ${ }^{15-20}$ cromatografia gasosa (GC) $)^{5,21,22}$ e cromatografia líquida de alta eficiência (HPLC). ${ }^{23}$ As vantagens e desvantagens de cada técnica analítica citada são discutidas por L. C. Côcco et al., 2005. ${ }^{24}$

Entre estas técnicas analíticas, a cromatografia gasosa tem sido extensamente utilizada pela indústria petroquímica, pois favorece uma melhor separação e resolução dos picos, principalmente em virtude do uso de softwares específicos que possibilitam uma identificação automática dos hidrocarbonetos presentes na composição da gasolina e também pela confiabilidade e precisão dos resultados, sua alta sensibilidade, baixo limite de detecção, baixo nível de ruído e ótimo fator de resposta. ${ }^{24}$ Por estas razões que a cromatografia gasosa com detector de ionização de chamas (GC-FID) consolidou-se como uma técnica padrão de análise para a determinação da composição química da gasolina.

Como ferramenta útil para auxiliar na interpretação dos dados obtidos no uso das técnicas mencionadas, tem sido empregado os métodos estatísticos multivariados de análise de dados..$^{10,11,25-28}$ Isto é verificado em vários trabalhos publicados recentemente, no que diz respeito à investigação sobre adulteração da gasolina com adição de diversos solventes orgânicos, e por outros tipos de combustíveis (diesel, álcool etílico anidro), além de estudos referentes à rastreabilidade e identificação de qual refinaria petrolífera supostamente a originou. , $, 5,8,9,21,25$

Os resultados de composição química cromatográfica e dos parâmetros físico-químicos das amostras de gasolina tipo A, obtidos neste trabalho, constituem dados multivariados que podem ser de difícil interpretação e, por isso, foram interpretados aplicando análise de componentes principais (PCA), como ferramenta quimiométrica, para melhor explorar a relação entre amostras e variáveis, possibilitando discriminar sua variabilidade de acordo com a similaridade apresentada pelas amostras coletadas em diferentes distribuidoras de combustíveis.

Para o desenvolvimento deste estudo objetivou-se: avaliar a eficácia da técnica analítica GC-FID equipada com o software Star DHA ${ }^{\mathrm{TM}}$ 
(Detailed Hydrocarbon Analysis), pois através da análise detalhada de hidrocarbonetos foi possível identificar e quantificar inúmeros compostos presentes nas amostras de gasolina tipo A; verificar as principais características físico-químicas deste combustível, através de ensaios segundo métodos oficiais (ASTM e ABNT); comparar os resultados obtidos com os limites estabelecidos pela ANP 57/2011. A análise de componentes principais foi utilizada para melhor interpretar os dados experimentais.

\section{PARTE EXPERIMENTAL}

\section{Instrumentação}

Análise por cromatografia gasosa com detector de ionização de chamas (GC-FID)

As análises cromatográficas foram realizadas em triplicata, utilizando um cromatógrafo a gás Varian, modelo CP 3800 (Walnut Creek, CA, USA) com detector de ionização de chamas e sistema de injeção split/splitless. Para injeção das amostras foi usado um amostrador automático Varian, modelo CP-8410. Foi utilizada, ainda, uma coluna capilar Petrocol ${ }^{\mathrm{TM}} \mathrm{DH} 100$ com dimensões de $100 \mathrm{~m}$ x $0.25 \mathrm{~mm}$ i.d x $0.5 \mu \mathrm{m}$ de espessura de filme (Supelco, Bellefonte, PA, USA). As temperaturas do injetor e detector foram mantidas constantes em $250{ }^{\circ} \mathrm{C}$ e $270{ }^{\circ} \mathrm{C}$, respectivamente. $\mathrm{O}$ aquecimento da coluna foi programado com gradiente de temperatura, inicialmente com $35^{\circ} \mathrm{C}$, isoterma de 15 minutos, aumento de $35{ }^{\circ} \mathrm{C}$ para $60{ }^{\circ} \mathrm{C}$ com taxa de aquecimento de $1{ }^{\circ} \mathrm{C} \mathrm{min}{ }^{-1}$, isoterma de 20 minutos, aumento de 60 ${ }^{\circ} \mathrm{C}$ até $200{ }^{\circ} \mathrm{C}$ com taxa de aquecimento de $2{ }^{\circ} \mathrm{C} \mathrm{min}{ }^{-1}$ e isoterma de 10 minutos, perfazendo um total de análise de 140 minutos. Para o controle instrumental do sistema cromatográfico e aquisição e tratamento dos dados foram utilizados os softwares Star Chromatography Workstation (versão 6.2) e Star DHA $A^{\mathrm{TM}}$ (versão 5.5), respectivamente.

\section{Parâmetros físico-químicos}

Todas as análises físico-químicas das amostras de gasolina tipo A foram medidas em triplicata, com exceção de cor e aspecto que foi realizada uma única vez. Para determinação de cor e aspecto, a análise foi realizada pelo método de inspeção visual. A determinação das temperaturas de destilação $T_{10 \%}, T_{50 \%}, T_{90 \%}$ (temperaturas equivalentes aos $10 \%, 50 \%$ e $90 \%$ do volume evaporado recuperado, respectivamente), além dos pontos iniciais e finais de ebulição $\left(T_{\mathrm{PIE}} \mathrm{e}\right.$ $T_{\mathrm{PFE}}$ ) e o resíduo de destilação foram obtidos utilizando um destilador automático atmosférico ISL, modelo AD86 5G2 (ASTM D86) ${ }^{29} \mathrm{~A}$ densidade foi determinada utilizando um densímetro automático digital KEM, modelo DA-500 (ASTM D 4052)..$^{30}$ Os parâmetros MON (Motor Octane Number), RON (Research Octane Number), IAD (MON+RON/2, Índice antidetonante) e as percentagens (\% v/v) de aromáticos, olefinas, hidrocarbonetos saturados e benzeno (ASTM D 6277) ${ }^{31}$ foram determinados utilizando um analisador portátil Petrospec, modelo GS 1000 PLUS com faixa espectral de $400-4000 \mathrm{~cm}^{-1}$.

\section{Padrão e solvente}

Para calibração do sistema cromatográfico foi utilizado padrão PIANO (Supelco, Bellefonte, PA, USA) de acordo com a ASTM D 6729. ${ }^{32}$ Para limpeza da micro-seringa de vidro e da linha de injeção do GC foi usada acetona (Tedia, Fairfield, OH, USA).

\section{Coleta e armazenagem das amostras}

Foi coletado um total de 24 amostras de gasolina tipo A de acordo com a ASTM D 4057, ${ }^{33}$ no período de março/abril/maio de 2008, em tanques pertencentes a oito diferentes companhias distribuidoras de combustíveis denominadas D1, D2, D3, D4, D5, D6, D7 e D8 existentes no Estado do Pará. As amostras foram acondicionadas em frascos de vidro apropriados, selados e lacrados e armazenadas sob refrigeração entre 5 a $13{ }^{\circ} \mathrm{C}$, para evitar a perda de compostos voláteis.

\section{Tratamento estatístico dos dados}

Para o tratamento estatístico dos resultados foram utilizados recursos básicos de estatística descritiva e a técnica quimiométrica análise de componentes principais (PCA), sendo processados com o auxílio do programa The Unscrambler 9.1 (CAMO S/A, USA). O nível de significância obtido para a análise estatística dos dados foi de $\mathrm{P}<0,05$.

Para a realização da PCA, primeiramente construiu-se uma matriz de dados ( $24 \times 11)$, na qual as amostras foram dispostas em linhas e as variáveis em colunas. Os dados originais foram autoescalonados, recurso utilizado em análise estatística onde se centra os dados na média e divide-se cada um pelo desvio padrão, de forma que todas variáveis passam a ter a mesma importância, ou seja, o mesmo peso. ${ }^{34,35} \mathrm{Em}$ seguida, os dados recalculados foram submetidos à PCA para melhor interpretação das 24 amostras de gasolina tipo A.

\section{Análise multivariada dos dados}

A análise da PCA consiste em reescrever as variáveis originais em novas variáveis denominadas componentes principais, a partir de uma transformação de coordenadas, com objetivo de simplificar as variações presentes em dados multivariados. ${ }^{35}$ Assim, a natureza multivariada dos dados pode ser projetada em um número reduzido de dimensões preservando ao mesmo tempo o máximo de informação e isto é feito através de cálculos de combinações lineares das variáveis originais, formando os componentes principais. Para isto, a matriz de dados originais é aproximada para duas matrizes menores (matrizes de escores e de pesos). ${ }^{36}$ Desta forma, como resultados da PCA são obtidos gráficos coordenados de dispersão bi ou tridimensionais de scores ou loadings, que facilita a visualização das informações dos dados experimentais. ${ }^{37,38}$

\section{RESULTADOS E DISCUSSÃO}

\section{Estudo da variabilidade da composição química da gasolina tipo A pelo método cromatográfico (GC-FID)}

Os resultados cromatográficos obtidos foram analisados no software Star $D H A^{\mathrm{TM}}$, que possui os tempos de retenção para cada composto da gasolina em seu banco de dados. Para a identificação e quantificação dos componentes da amostra procedeu-se à comparação entre os tempos de retenção e área integrada para cada um dos compostos. Uma das dificuldades observadas é que apesar de reconhecer a maioria dos constituintes, existe uma quantidade de compostos presentes na gasolina que ainda não podem ser identificadas pelo software Star $D H A^{\mathrm{TM}}$. Normalmente, os compostos não identificados (desconhecidos) são aqueles que contêm nitrogênio e enxofre, porém, existem algumas olefinas e aromáticos que o software Star $D H A^{\mathrm{TM}}$ também não reconhece. ${ }^{24}$ Assim, a lista de composição química reporta, além da classe de hidrocarbonetos principais (parafinas, isoparafinas, naftenos, olefinas e aromáticos) o percentual de compostos desconhecidos (não identificados).

O cromatograma observado na Figura 1 obtido para a gasolina tipo A foi dividido em três regiões. De acordo com o estudo realizado por L. S. M. Wiedemann et al.; 2003 e $2005,{ }^{5,7}$ na primeira 


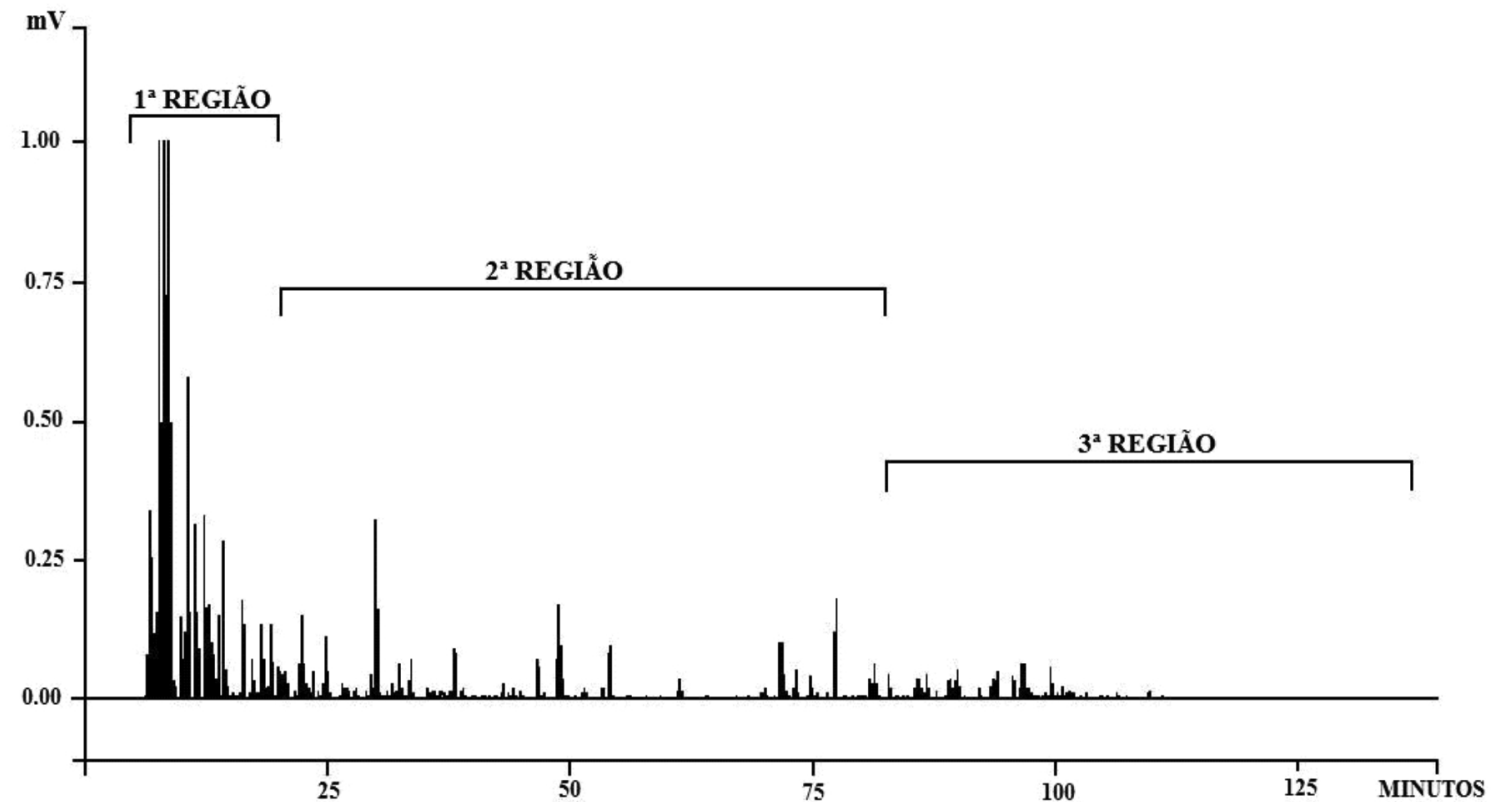

Figura 1. Perfil cromatográfico da gasolina tipo A de acordo com ASTM 6729

região do cromatograma estão situados os picos mais intensos, referentes aos hidrocarbonetos alifáticos leves $\left(\mathrm{nC}_{4}-\mathrm{nC}_{6}\right) \operatorname{com} \mathrm{T}_{\mathrm{R}}$ entre 0-20 minutos de análise, que são compostos majoritários, onde se encontram as parafinas, isoparafinas, naftenos e olefinas de baixo ponto de ebulição. Na segunda região do cromatograma estão situados os picos de intensidade intermediária, referente aos hidrocarbonetos aromáticos $\left(\mathrm{nC}_{7}-\mathrm{nC}_{9}\right)$ com $\mathrm{T}_{\mathrm{R}}$ entre 20-80 minutos de análise. Na última região do cromatograma, referente aos hidrocarbonetos alifáticos pesados $\left(\mathrm{nC}_{10}-\mathrm{nC}_{13}\right)$, encontram-se os picos de menor intensidade com $\mathrm{T}_{\mathrm{R}}$ entre 80-140 minutos de análise, que são os compostos minoritários, onde se encontram as parafinas, isoparafinas, naftenos e olefinas de elevado ponto de ebulição, e também a maior quantidade de compostos considerados desconhecidos pelo $D H A^{\mathrm{TM}}$. Na Tabela $1 \mathrm{~S}$ (material suplementar) estão apresentados os resultados da composição química para cada classe de hidrocarbonetos referente as 24 amostras analisadas pelo método cromatográfico.

Para cada amostra analisada em triplicata, o \%RSD calculado para os compostos individuais foi inferior a $4 \%$, enquanto para as classes de hidrocarbonetos os valores variaram de $0,11 \%$ a $1,65 \%$. Um total de 222 compostos da gasolina tipo A foi identificado e os 20 mais abundantes com seus respectivos $\mathrm{T}_{\mathrm{R}}$ estão apresentados na Tabela 1 .

Para observar a variabilidade da composição química da gasolina tipo A, as classes de hidrocarbonetos foram monitoradas por meio dos dados de estatística descritiva (média global \% (v/v), \%SD e \% RSD) obtidos no período de março/abril/maio de 2008 para as 24 amostras de gasolina tipo A. Os resultados da composição química foram expressos em termos das classes de hidrocarbonetos, que estão apresentados na Tabela 2.

Os valores de \%RSD apresentados na Tabela 2 revelaram variações na composição química das amostras de gasolina tipo A, decorrentes principalmente da natureza do petróleo e de seu processo de produção. ${ }^{2,3}$ Estes resultados mostraram que a quantidade de compostos isoparafínicos e olefínicos presentes na composição química são majoritários em relação às outras classes de hidrocarbonetos, $23,3 \%$ e $22,9 \%$, respectivamente, seguidos pelos aromáticos $18,9 \%$ e em menor quantidade os compostos naftênicos e parafínicos, $11.6 \%$ e $11,3 \%$, respectivamente, o que é esperado para gasolina tipo A produzida no Brasil. ${ }^{1,3}$
Tabela 1. Hidrocarbonetos com maiores médias de concentração \% (v/v) nas amostras de gasolina tipo A e seus respectivos $\mathrm{T}_{\mathrm{R}}(\mathrm{min})$

\begin{tabular}{lcc}
\hline Compostos & $\%(\mathrm{v} / \mathrm{v})$ & $\mathrm{T}_{\mathrm{R}}$ \\
\hline Isopentano & 4,73 & 7,210 \\
n-Pentano & 4,13 & 7,986 \\
2-Metil-2-buteno & 3,65 & 8,455 \\
2-Metilpentano & 3,25 & 10,535 \\
Tolueno & 3,24 & 29,839 \\
m-Xileno & 2,71 & 48,753 \\
n-Hexano & 2,67 & 12,136 \\
Trans-2-penteno & 2,57 & 8,112 \\
2-Metil-1-buteno & 2,18 & 7,428 \\
Metilciclopentano & 2,12 & 14,423 \\
n-Heptano & 2,10 & 22,325 \\
1,2,4-Trimetilbenzeno & 2,03 & 77,451 \\
n-Octano & 1,86 & 37,982 \\
1,1,2-Trimetilciclohexano & 1,81 & 50,136 \\
Metilciclohexano & 1,78 & 23,620 \\
o-Xileno & 1,67 & 54,065 \\
3-Metilpentano & 1,65 & 6,374 \\
1-Metilciclopentano & & 14,884 \\
p-Xileno & 1,61 & \\
n-Butano & 3962 \\
\hline
\end{tabular}

\section{Avaliação dos parâmetros físico-químicos}

A Tabela 3 apresenta os resultados estatísticos descritivos, como média, \%SD e valores mínimos e máximos, para melhor avaliar os parâmetros físico-químicos estudados no período de março/abril/ maio de 2008 para as 24 amostras de gasolina tipo A.

Os resultados obtidos dos parâmetros fisíco-químicos foram comparados com os limites máximos e mínimos estabelecidos pela 
Tabela 2. Composição química das classes de hidrocarbonetos obtidas pelo método cromatográfico nos meses de março/abril/maio de 2008 para as amostras de gasolina tipo A $(n=3)$

\begin{tabular}{lccc}
\hline $\begin{array}{l}\text { Classe de } \\
\text { hidrocarbonetos }\end{array}$ & $\%(\mathrm{v} / \mathrm{v})^{\mathrm{a}} \pm \mathrm{SD}$ & $\%$ RSD & $\begin{array}{c}\text { Mínimo-Máximo } \\
\%(\mathrm{v} / \mathrm{v})\end{array}$ \\
\hline Aromáticos & $18,9 \pm 1,10$ & 5,8 & $17,0-21,5$ \\
Isoparafinas & $23,3 \pm 1,85$ & 7,7 & $20,1-25,7$ \\
Naftenos & $11,6 \pm 1,75$ & 14,6 & $9,9-16,1$ \\
Olefinas & $22,9 \pm 2,50$ & 10,9 & $16,9-25,8$ \\
Parafinas & $11,3 \pm 1,75$ & 15,0 & $9,2-14,5$ \\
Total & $88,1 \pm 0,60$ & 0,7 & $87,1-89,4$ \\
Desconhecidos & $11,8 \pm 0,63$ & 5,3 & $10,5-12,9$ \\
\hline
\end{tabular}

$\mathrm{SD}=$ desvio padrão; RSD = desvio padrão relativo; ${ }^{a}$ Média das 24 amostras de gasolina tipo A com nível de confiança de $95 \%$.

ANP, e todas as amostras comportaram-se dentro dos limites, sendo consideradas conforme em termos de qualidade. Os resultados físico-químicos das 24 amostras de gasolina tipo A, coletadas para este trabalho, estão apresentadas na Tabela 2S (material suplementar).

\section{Análise de componentes principais (PCA)}

Para melhor extração de informações das amostras e das variáveis e uma avaliação mais minuciosa dos dados, aplicou-se a análise de componentes principais (PCA) a fim de observar semelhanças e/ ou diferenças entre as amostras de gasolina tipo A, a partir de suas características físico-químicas $\left(T_{10 \%}, T_{50 \%}, T_{90 \%}, T_{\mathrm{PFE}}, \mathrm{MON}, \mathrm{IAD}\right) \mathrm{e}$ composição cromatográfica referente às classes de hidrocarbonetos (parafinas, isoparafinas, aromáticos, naftenos e olefinas).
Assim, a PCA projetou os dados em um espaço com 11 dimensões no plano (11 PCs), sendo que de acordo com o critério de Kaiser, somente as três primeiras componentes foram mais significativas para a variância explicada dos dados, pois cada componente apresentou autovalores $>1,{ }^{34,36}$ sendo que, combinadas, explicou $94,7 \%$ da variância total dos dados originais. Os pesos dos coeficientes referentes aos loadings para as três componentes principais mais significativas estão apresentados na Tabela 4.

Na Figura 2, através da projeção da PC1 x PC2, observou-se claramente no gráfico dos scores a formação de quatro agrupamentos, denominados de grupos A, B, C e D. O grupo A foi formado exclusivamente pelas amostras 1, 9 e 17 (D1). $O$ grupo B foi formado por 15 amostras, subdivididas da seguinte forma: amostras 4, 12 e 20 (D4), amostras 5, 13 e 21 (D5), amostras 6, 14 e 22 (D6), amostras 7, 15 e 23 (D7) e amostras 8, 16 e 24 (D8). O grupo C foi composto pelas amostras 2, 10 e 18 (D2) e o grupo D pelas amostras 3, 11 e 19 (D3).

Através da distribuição das amostras no plano bidimensional verificaram-se semelhanças entre as amostras pertencentes aos grupos A e B posicionados positivamente na $\mathrm{PC} 1$, com exceção das amostras da distribuidora D7, ocorrendo a mesma situação com as amostras pertencentes aos grupos C e D posicionados positivamente na PC2, com exceção da amostra 19 da distribuidora D3. Acredita-se que a formação desses grupos, independente do mês que as amostras foram coletadas, foi atribuída a particularidades do processo de produção utilizado pela refinaria que a originou.

Na Figura 3, pode ser observado no gráfico dos loadings, através da projeção da PC1 x PC2 (Figura 3a) e PC1 x PC3 (Figura 3b), quais as variáveis com maior efeito na separação das amostras.

A primeira componente (PC1) foi a que mais contribuiu para separação das amostras, explicando $56,2 \%$ da variância dos dados, concentrando informações a respeito das variáveis $T_{90 \%}(0,478)$, IAD $(0,105)$, aromáticos $(0,177)$ e olefinas $(0,557)$, sendo as mais

Tabela 3. Média \pm DP e valores mínimos e máximos dos parâmetros físico-químicos monitorados no período de março/abril/maio de 2008 nas amostras de gasolina tipo $\mathrm{A}(n=3)$

\begin{tabular}{|c|c|c|c|c|c|c|}
\hline \multirow[b]{2}{*}{ Parâmetros } & \multicolumn{2}{|c|}{ Março } & \multicolumn{2}{|c|}{ Abril } & \multicolumn{2}{|c|}{ Maio } \\
\hline & $\%(\mathrm{v} / \mathrm{v}) \pm \mathrm{SD}$ & $\begin{array}{c}\text { Mínimo-Máximo } \\
\text { \% (v/v) }\end{array}$ & $\%(\mathrm{v} / \mathrm{v}) \pm \mathrm{SD}$ & $\begin{array}{c}\text { Mínimo-Máximo } \\
\%(\mathrm{v} / \mathrm{v})\end{array}$ & $\%(\mathrm{v} / \mathrm{v}) \pm \mathrm{SD}$ & $\begin{array}{c}\text { Mínimo-Máximo } \\
\% \text { (v/v) }\end{array}$ \\
\hline Densidade a $20{ }^{\circ} \mathrm{C}\left(\mathrm{g} / \mathrm{cm}^{3}\right)$ & $0,7441 \pm 0,002$ & $0,7411-0,7465$ & $0,7442 \pm 0,003$ & $0,7408-0,7476$ & $0,7452 \pm 0,002$ & $0,7419-0,7481$ \\
\hline \multicolumn{7}{|l|}{ Destilação $\left({ }^{\circ} \mathrm{C}\right)$} \\
\hline$T_{\mathrm{PIE}}$ & $35,5 \pm 1,25$ & $34,1-37,8$ & $36,1 \pm 1,22$ & $34,8-38,5$ & $35,8 \pm 1,03$ & $34,6-38,0$ \\
\hline$T_{10 \%}$ & $55,4 \pm 1,11$ & $53,7-56,9$ & $55,0 \pm 0,69$ & $54,2-56,3$ & $55,2 \pm 1,07$ & $53,1-56,6$ \\
\hline$T_{50 \%}$ & $103,8 \pm 1,53$ & $101,2-103,8$ & $104,2 \pm 1,37$ & $102,1-105,8$ & $104,8 \pm 1,60$ & $101,7-106,4$ \\
\hline$T_{90 \%}$ & $176,2 \pm 3,35$ & $171,3-176,2$ & $175,2 \pm 4,13$ & $171,4-184,1$ & $175,2 \pm 4,08$ & $170,3-183,5$ \\
\hline$T_{\mathrm{PFE}}$ & $208,3 \pm 3,98$ & $204,8-217,0$ & $206,6 \pm 2,32$ & $203,3-209,7$ & $206,6 \pm 2,43$ & $204,1-211,0$ \\
\hline Resíduo (\% v/v) & $1,3 \pm 0,19$ & $1,1-1,7$ & $1,2 \pm 0,07$ & $1,1-1,3$ & $1,2 \pm 0,11$ & $1,1-1,4$ \\
\hline MON & $80,6 \pm 1,10$ & $79,1-81,9$ & $80,5 \pm 1,19$ & $79,1-81,8$ & $80,6 \pm 1,11$ & $79,1-81,8$ \\
\hline RON & $91,2 \pm 1,53$ & $88,1-91,2$ & $90,8 \pm 1,24$ & $88,3-92,4$ & $90,9 \pm 1,40$ & $88,1-92,5$ \\
\hline IAD & $85,9 \pm 1,11$ & $83,8-86,9$ & $85,7 \pm 1,04$ & $83,8-86,7$ & $85,7 \pm 1,03$ & $84,0-86,7$ \\
\hline Benzeno (\% v/v) & $0,78 \pm 0,10$ & $0,65-0,96$ & $0,74 \pm 0,08$ & $0,62-0,84$ & $0,75 \pm 0,07$ & $0,66-0,90$ \\
\hline \multicolumn{7}{|l|}{ Hidrocarbonetos (\% v/v) } \\
\hline Aromáticos & $20,3 \pm 3,18$ & $13,1-23,2$ & $20,5 \pm 3,91$ & $13,3-23,19$ & $20,9 \pm 3,17$ & $13,6-24,2$ \\
\hline Olefínas & $30,0 \pm 5,51$ & $24,5-39,8$ & $29,0 \pm 3,91$ & $25,8-35,1$ & $28,8 \pm 4,62$ & $23,1-36,3$ \\
\hline Saturados & $48,9 \pm 5,40$ & $40,4-55,1$ & $49,6 \pm 4,79$ & $41,4-56,4$ & $49,5 \pm 5,42$ & $41,2-56,4$ \\
\hline
\end{tabular}

MON = Motor Octane Number; RON = Research Octane Number; IAD = Índice antidetonante.Valores máximos para temperatura de destilação para $T_{10 \%}$ $\left(65^{\circ} \mathrm{C}\right), T_{50 \%}\left(120^{\circ} \mathrm{C}\right), T_{90 \%}\left(155-190^{\circ} \mathrm{C}\right)$ e $T_{\mathrm{PFE}}\left(220^{\circ} \mathrm{C}\right)$; resíduo $(2,0 \% \mathrm{v} / \mathrm{v})$; benzeno $(1,2 \% \mathrm{v} / \mathrm{v})$, para hidrocarbonetos aromáticos $(57 \% \mathrm{v} / \mathrm{v}) ;$ olefinas $(38 \%$ v/v); e valores mínimos para MON $(80,0)$ e IAD $(85,0)$. 
Tabela 4. Loadings para as três componentes principais mais significativas, autovalores e variância explicada (\%) para matriz de 24 x 11 . Os valores mais significativos estão em negrito

\begin{tabular}{lccc}
\hline Variáveis & PC1 & PC2 & PC3 \\
\hline$T_{10 \%}$ & $-0,248$ & $-0,170$ & $-0,041$ \\
$T_{50 \%}$ & $-0,092$ & $\mathbf{- 0 , 3 0 8}$ & $\mathbf{- 0 , 4 0 9}$ \\
$T_{90 \%}$ & $\mathbf{0 , 4 7 8}$ & 0,259 & $-0,238$ \\
$T_{\text {PFE }}$ & $-0,124$ & $\mathbf{0 , 7 5 6}$ & $\mathbf{0 , 3 5 1}$ \\
MON & 0,022 & $-0,264$ & 0,151 \\
IAD & 0,105 & $-0,161$ & 0,161 \\
Parafinas & $\mathbf{- 0 , 3 8 2}$ & 0,123 & $\mathbf{- 0 , 3 0 8}$ \\
Isoparafinas & $-0,054$ & $\mathbf{- 0 , 3 2 0}$ & $\mathbf{0 , 6 7 5}$ \\
Aromáticos & 0,177 & 0,114 & $-0,153$ \\
Naftenos & $\mathbf{- 0 , 4 2 9}$ & 0,034 & $-0,157$ \\
Olefinas & $\mathbf{0 , 5 5 7}$ & $-0,098$ & $-0,058$ \\
Autovalores & 5,42 & 2,31 & 1,53 \\
Variância explicada (\%) & 56,2 & 28,4 & 10,1 \\
Variância acumulada (\%) & 56,2 & 84,6 & 94,7 \\
\hline
\end{tabular}

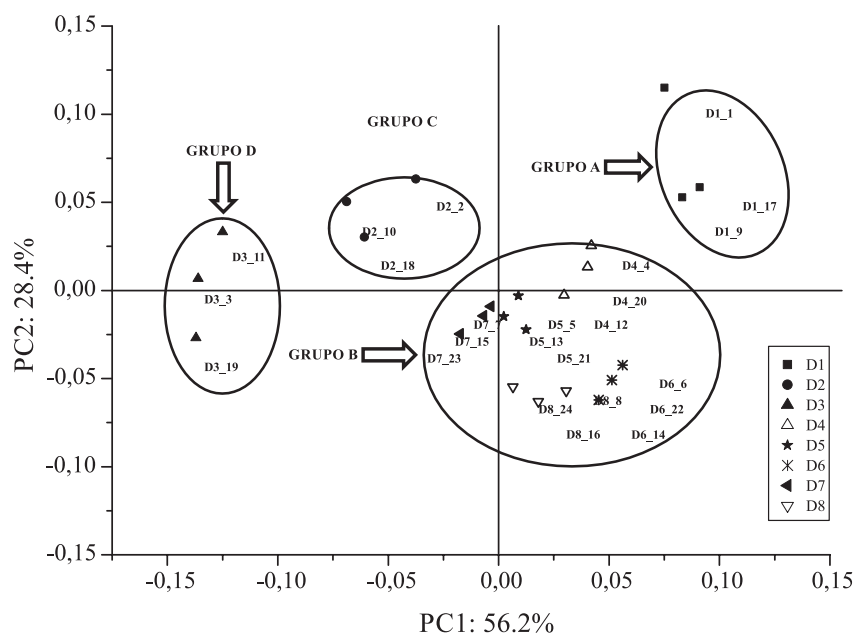

Figura 2. Gráfico dos Scores PC1 x PC2 correspondentes as 24 amostras de gasolina tipo $A$

significativas para a formação dos grupos A e B, indicando que as variáveis $T_{90 \%}$ e olefinas apresentaram correlação significativa com as amostras do grupo $\mathrm{A}$, enquanto que IAD e aromáticos foram responsáveis para formação do grupo $\mathrm{B}$, apresentando ainda uma correlação negativa com as variáveis naftenos $(-0,429)$ e parafinas $(-0,382)$ proporcionando a formação dos grupos $\mathrm{C}$ e $\mathrm{D}$.

A segunda componente (PC2) foi responsável por 28,4\% da variância dos dados, concentrando informações a respeito das variáveis $T_{90 \%}(0,259), T_{\mathrm{PFE}}(0,756)$, parafinas $(0,123)$ e aromáticos $(0,114)$, que apresentaram correlação positiva, sendo que a variável $T_{\mathrm{PFE}}$ foi mais significativa para a discriminação das amostras pertencentes ao grupo $\mathrm{C}$, enquanto as variáveis parafinas e naftenos foram mais importantes para a discriminação das amostras do grupo D, apresentando ainda uma correlação negativa significativa para o $T_{50 \%}(-0,308)$ e isoparafinas $(-0,320)$.

A terceira componente (PC3) explicou $10,1 \%$ da variância dos dados, concentrou informações a respeito das variáveis $T_{\mathrm{PFE}}(0,351)$ e isoparafinas $(0,675)$, que apresentaram correlação positiva, sendo que a variável isoparafinas foi mais significativa para formação do
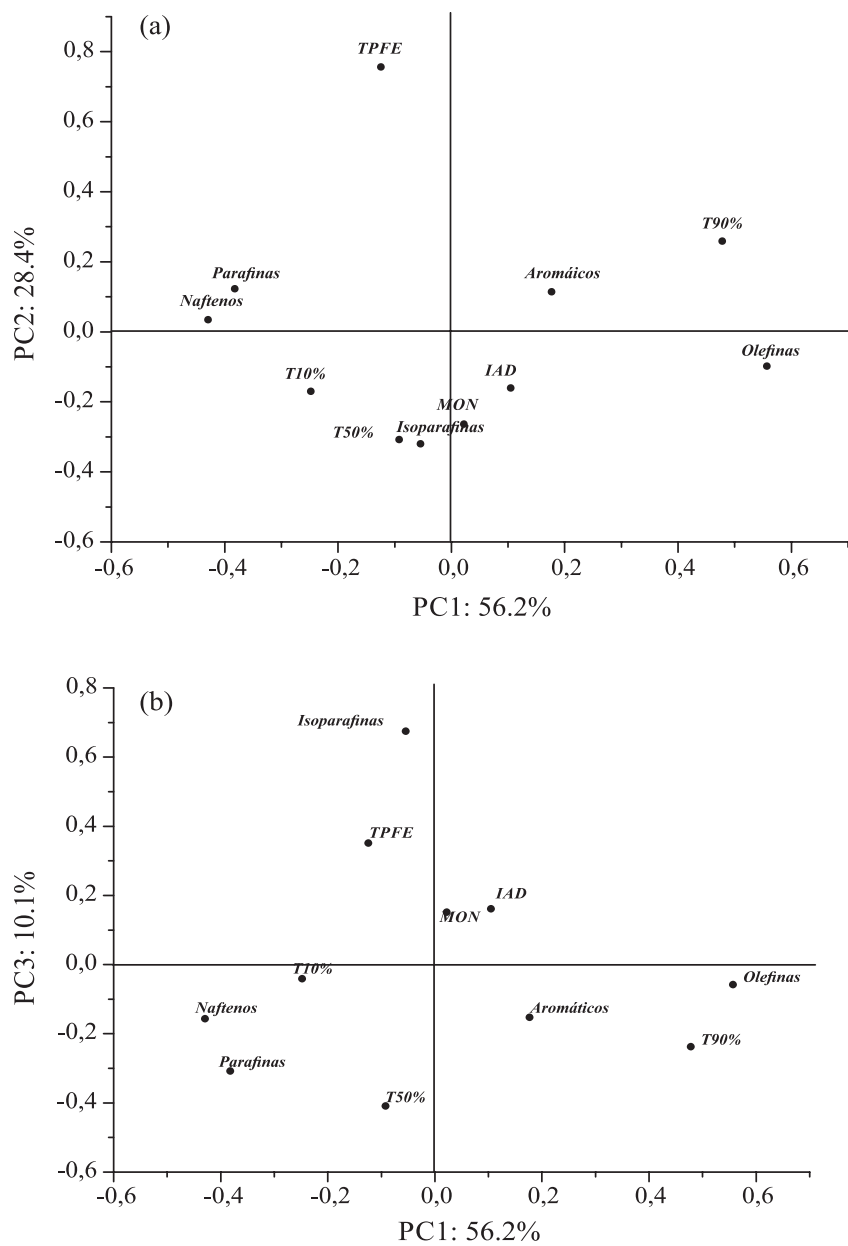

Figura 3. Gráfico dos Loadings correspondentes aos 11 parâmetros estudados nas 24 amostras de gasolina Tipo A: PC1 x PC2 (a) e PC1 x PC3 (b)

grupo $\mathrm{C}$, e uma correlação negativa significativa para as variáveis $T_{50 \%}(-0,409)$ e parafinas $(-0,308)$.

\section{CONCLUSÃO}

Por meio do estudo da variabilidade da composição química cromatográfica realizada nas 24 amostras utilizadas neste trabalho foi possível detectar pequenas variações em sua composição química no período de três meses e quantificar aproximadamente 222 compostos, correspondendo a um volume total de $88,1 \%$ (v/v) de compostos identificados. Em relação aos parâmetros físico-químicos, os resultados revelaram que $100 \%$ das amostras atenderam os limites estabelecidos pela legislação brasileira para a gasolina tipo A conforme a ANP/2011.

Com relação ao modelo obtido com a análise de componentes principais (PCA), este se mostrou eficaz na interpretação das amostras de gasolina tipo A, considerando os dados analisados. Desta forma, comparando-se os gráficos dos scores com dos loadings obtidos da combinação da PC1 x PC2, observou-se a separação das 24 amostras em 4 grupos, sendo que as variáveis mais significativas para esta separação foram $T_{50 \%}, T_{90 \%}, T_{\mathrm{PFE}}$, isoparafinas, parafinas, naftenos e olefinas. Assim, as informações extraídas através da PCA permitiu observar diferenças e similaridades entre as amostras coletadas nas oito distribuidoras de combustíveis no Estado do Pará.

\section{AGRADECIMENTOS}

Os autores agradecem a Coordenação de Aperfeiçoamento de 
Pessoal de Nível Superior (CAPES) pela bolsa de estudo concedida a F. I. M. Carvalho, a Transpetro S. A. pelo fornecimento das amostras de gasolina tipo A utilizadas neste estudo e ao Laboratório de Pesquisa e Análise de Combustíveis (LAPAC, UFPA) cedendo sua infra-estrutura para realização das análises.

\section{MATERIAL SUPLEMENTAR}

As Tabelas $1 \mathrm{~S}$ e $2 \mathrm{~S}$ apresentam resultados da composição química cromatográfica e as análises físico-químicas das 24 amostras de gasolina tipo A, respectivamente.

\section{REFERÊNCIAS}

1. Ministério das Minas de Energia (MME); Balanço energético Nacional. Disponível em: https://ben.epe.gov.br/downloads/Resultados_Pre_ BEN_2012.pdf, acessado em Fevereiro 2013.

2. Takeshita, E. V.; Resende, R. V. P.; Guelli, U.; de Souza, S. M. A.; Ulson de Souza, A. A.; Fuel 2008, 87, 2168.

3. Pasadakis, N.; Gaganis, V.; Foteinopoulos, C.; Fuel Process. Technol. 2006, 87, 505.

4. Oliveira, F. S.; Teixeira, L. S. G.; Araújo, M. C. U.; Korn, M.; Fuel 2004, 83, 917.

5. Wiedemann, L. S. M.; d'Avila, L. A.; Azevedo, D. A.; Chromatographia 2003, 58, 501.

6. Agência Nacional do Petróleo (ANP). Available: http//www.anp.gov.br, acessado em Novembro 2012.

7. Wiedemann, L. S. M.; d'Avila, L. A.; Azevedo, D. A.; Fuel 2005, 84, 467.

8. Pereira, R. C. C.; Skrobot, V. L.; Castro, E. V. R.; Fortes, I. C. P.; Pasa, V. M. D.; Energy Fuels 2006, 20, 1097.

9. Teixeira, L. S. G.; Oliveira, F. S.; Santos, H. C.; Cordeiro, P. W. L.; Almeida, S. Q.; Fuel 2008, 87, 346.

10. Honorato, F. A; Barros Neto, B.; Pimentel, M. F.; Stragevitch, L.; Galvão, R. K. H.; Fuel 2008, 87, 3706.

11. Balabin, R. M.; Safieva, R. Z.; Fuel 2008, 87, 1096.

12. Mittermayr, C. R.; Drouen, A. C. J. H.; Otto, M.; Grasserbauer, M.; Anal. Chim. Acta 1994, 294, 227.

13. Wienke, D.; Kateman, G.; Chemometrics and Intelligent Laboratory Systems 1994, 23, 309.

14. Eghbaldar, A.; Forrest, T. P.; Cabrol-Bass, D.; Anal. Chim. Acta 1998, 359, 283.

15. Kalsi, W. R.; Sarpal, A. S.; Jain, S. K.; Srivastava, S. P.; Bhatnagar, A. K.; Energy Fuels 1995, 9, 574.

16. Sarpal, A. S.; Kapur, G. S.; Mukherjee, S.; Jain, S. K.; Energy Fuels 1997, 11, 662.

17. Sarpal, A. S.; Kapur, G. S.; Singh, A. P.; Fuel 2000, 79, 1023.
18. Sarpal, A. S.; Kapur, G. S.; Mukherjee, S.; Tiwari, A. K.; Fuel 2001, 80 , 521.

19. Meusinger, R.; Moros, R.; Fuel 2001, 80, 613.

20. Kaiser, C. R.; Borges, J. L.; Santos, A. R.; Azevedo, D. A.; d'Avila, L. A.; Fuel 2010, 89, 99.

21. Skrobot, V. S.; Castro, E. V. R.; Pereira, R. C. C.; Pasa, V. M. D.; Fortes, I. C. P.; Energy Fuels 2005, 19, 2350.

22. Ré-Poppi, N.; Almeida, F. F. P.; Cardoso, C. A. L.; Raposo Jr., J. L.; Viana, L. H.; Silva, T. Q.; Fuel 2009, 88, 418.

23. Smits, J. R. M.; Melssen, W. J.; Daalmans, G. J.; Kateman, G.; Comput. Chem. 1994, 18, 157.

24. Côcco, L. C.; Yamamoto, C. I.; von Meien, O. F.; Chemometrics and Intelligent Laboratory Systems 2005, 76, 55.

25. Skrobot, V. S.; Castro, E. V. R.; Pereira, R. C. C.; Pasa, V. M. D.; Fortes, I. C. P.; Energy Fuels 2007, 21, 3394.

26. Morris, R. E.; Hammond, M. H.; Shafer, R. E.; Gardner, W. P.; RosePehrsson, S. L.; Energy Fuels 2004, 18, 485.

27. Barbeira, P. J. S.; Pereira, R. C. C.; Corgozinho, C. N. C.; Energy Fuels 2007, 21, 2212.

28. Aleme, H. G.; Costa, L. M.; Barbeira, P. J. S.; Fuel 2008, 87, 3664.

29. American Society for Testing and Material - ASTM D4057-06: Standard practice for manual sampling of petroleum and petroleum products, Annual Books of ASTM Standard, vol. 05, 2002.

30. American Society for Testing and Material - ASTM D86: Standard test method for distillation of petroleum products at atmospheric pressure, 1995.

31. American Society for Testing and Material - ASTM D4052: Standard test method for density and relative density of liquids by digital density meter, 1986.

32. American Society for Testing and Material - ASTM D6277: Standard test method for determination of benzene in spark-ignition engine fuels using Mid Infrared Spectroscopy, 1998.

33. American Society for Testing and Material - ASTM D6729: Standard test method for determination of individual components in spark ignition engine fuels by 100 meter capillary high resolution gas chromatography, 2002.

34. Vandeginste, B. G. M.; Massart, D. L.; Buydens, L. M. C.; De Jong, S.; Lewi, P. J.; Smeyers-Verbeke, J.; Handbook of Chemometrics and Qualimetrics: Part b, Elsevier: Amsterdam, 1998.

35. Mingoti, S. A.; Análise de Dados através de Métodos de Estatística Multivariada, $1^{\text {st }}$ ed., Editora UFMG: Belo Horizonte, 2005.

36. Beebe, K. R.; Pell, R. J.; Seasholtz, M. B.; Chemometrics: A Pratical Guide, John Wiley \& Sons: New York, 1998.

37. Sharaf, M. A.; Illman, D. L.; Kowalski, B. R.; Chemometrics, $1^{\text {a }}$ ed., John Wiley: New York, 1986

38. Barros Neto, B.; Scarminio, I. S.; Bruns, R. E.; Quim. Nova 2006, 29 , 1401. 


\section{ESTUDO DA QUALIDADE DA GASOLINA TIPO A E SUA COMPOSIÇÃO QUÍMICA EMPREGANDO ANÁLISE DE COMPONENTES PRINCIPAIS}

Fábio Israel M. Carvalho* e Heronides A. Dantas Filho

Faculdade de Química, Instituto de Ciências Exatas e Naturais, Universidade Federal do Pará, 66075-110 Belém - PA, Brasil

Tabela 1S. Composição química cromatográfica de todas as amostras de gasolina tipo A analisadas $(n=3)$

\begin{tabular}{|c|c|c|c|c|c|c|c|c|}
\hline Distribuidora & $\mathrm{N}^{\circ}$ Amostras & $\begin{array}{c}\text { Parafinas } \\
(\% \mathrm{v} / \mathrm{v})\end{array}$ & $\begin{array}{c}\text { Isoparafinas } \\
(\% \mathrm{v} / \mathrm{v})\end{array}$ & $\begin{array}{c}\text { Aromáticos } \\
(\% \mathrm{v} / \mathrm{v})\end{array}$ & $\begin{array}{l}\text { Naftenos } \\
(\% \mathrm{v} / \mathrm{v})\end{array}$ & $\begin{array}{l}\text { Olefinas } \\
(\% \mathrm{v} / \mathrm{v})\end{array}$ & $\begin{array}{l}\text { Total } \\
(\% \mathrm{v} / \mathrm{v})\end{array}$ & $\begin{array}{c}\text { Desconhecidos } \\
(\% \mathrm{v} / \mathrm{v})\end{array}$ \\
\hline \multicolumn{9}{|l|}{ Março } \\
\hline D1 & 1 & 9,5 & 20,4 & 21,5 & 10,3 & 25,8 & 87,5 & 12,5 \\
\hline D2 & 2 & 12,5 & 23,9 & 18,6 & 12,1 & 21,7 & 88,8 & 11,1 \\
\hline D3 & 3 & 14,5 & 23,5 & 17,2 & 15,6 & 17,8 & 88,6 & 11,3 \\
\hline D4 & 4 & 9,2 & 25,5 & 18,1 & 10,7 & 24,3 & 87,8 & 12,0 \\
\hline D5 & 5 & 12,1 & 20,7 & 19,7 & 11,5 & 25,1 & 89,1 & 10,8 \\
\hline D6 & 6 & 9,5 & 24,8 & 19,5 & 10,3 & 24,8 & 88,9 & 11,0 \\
\hline D7 & 7 & 12,2 & 23,8 & 18,6 & 11,9 & 21,7 & 88,2 & 11,7 \\
\hline D8 & 8 & 9,5 & 25,2 & 18,7 & 10,3 & 23,9 & 87,6 & 12,3 \\
\hline \multicolumn{9}{|l|}{ Abril } \\
\hline D1 & 9 & 11,1 & 20,1 & 20,5 & 10,8 & 24,6 & 87,1 & 12,9 \\
\hline D2 & 10 & 12,9 & 23,6 & 19,3 & 12,0 & 20,6 & 88,4 & 11,5 \\
\hline D3 & 11 & 14,3 & 23,3 & 17,3 & 15,4 & 16,9 & 87,2 & 12,8 \\
\hline D4 & 12 & 9,6 & 25,7 & 18,6 & 10,3 & 23,6 & 87,8 & 12,1 \\
\hline D5 & 13 & 12,6 & 20,3 & 19,9 & 11,7 & 24,9 & 89,4 & 10,5 \\
\hline D6 & 14 & 9,3 & 24,3 & 19,2 & 10,7 & 24,6 & 88,1 & 11,7 \\
\hline D7 & 15 & 12,6 & 23,4 & 18,3 & 11,6 & 22,2 & 88,1 & 11,8 \\
\hline D8 & 16 & 9,2 & 25,6 & 18,4 & 10,7 & 24,3 & 88,2 & 11,7 \\
\hline \multicolumn{9}{|l|}{ Maio } \\
\hline D1 & 17 & 10,4 & 21,5 & 20,7 & 10,1 & 25,1 & 87,8 & 12,1 \\
\hline D2 & 18 & 12,1 & 22,7 & 18,4 & 12,8 & 21,5 & 87,5 & 12,4 \\
\hline D3 & 19 & 13,9 & 23,0 & 17,0 & 16,1 & 17,8 & 87,8 & 12,1 \\
\hline D4 & 20 & 10,0 & 25,4 & 18,5 & 9,9 & 23,9 & 87,7 & 12,3 \\
\hline D5 & 21 & 12.0 & 20,7 & 19,3 & 11,1 & 25,8 & 88,9 & 11,1 \\
\hline D6 & 22 & 9,7 & 24,8 & 19,3 & 10,2 & 24,2 & 88,2 & 11,6 \\
\hline D7 & 23 & 12,7 & 22,7 & 18,9 & 12,1 & 21,8 & 88,2 & 11,6 \\
\hline D8 & 24 & 9,8 & 25,3 & 18,7 & 11,0 & 23,7 & 88,5 & 11,5 \\
\hline
\end{tabular}

*e-mail: fabioimc@yahoo.com.br 
Tabela 2S. Parâmetros físico-químicos de todas as amostras de gasolina tipo A analisadas $(n=3)$

\begin{tabular}{|c|c|c|c|c|c|c|c|c|c|c|c|c|}
\hline \multirow{2}{*}{$\begin{array}{l}\text { Distribui- } \\
\text { dora }\end{array}$} & \multirow{2}{*}{$\begin{array}{c}\mathrm{N}^{\mathrm{o}} \\
\text { Amostras }\end{array}$} & \multirow{2}{*}{$\begin{array}{c}\text { Densidade } \\
\left(\mathrm{g} / \mathrm{cm}^{3}\right)\end{array}$} & \multicolumn{4}{|c|}{ Temperaturas de destilação $\left({ }^{\circ} \mathrm{C}\right)$} & \multirow{2}{*}{$\begin{array}{c}\text { Resíduo } \\
(\% \text { v/v) }\end{array}$} & \multirow{2}{*}{$\begin{array}{c}\text { Benzeno } \\
(\% \mathrm{v} / \mathrm{v})\end{array}$} & \multirow{2}{*}{$\begin{array}{c}\text { Aromáticos } \\
(\% \mathrm{v} / \mathrm{v})\end{array}$} & \multirow{2}{*}{$\begin{array}{l}\text { Olefinas } \\
(\% \mathrm{v} / \mathrm{v})\end{array}$} & \multirow[t]{2}{*}{ MON } & \multirow[t]{2}{*}{$\mathrm{IAD}$} \\
\hline & & & $T_{10 \%}$ & $T_{50 \%}$ & $T_{90 \%}$ & $T_{\mathrm{PFE}}$ & & & & & & \\
\hline \multicolumn{13}{|l|}{ Março } \\
\hline D1 & 1 & 0,7453 & 53,7 & 104,8 & 182,0 & 217,0 & 1,7 & 0,76 & 19,0 & 39,8 & 80,9 & 86,9 \\
\hline D2 & 2 & 0,7428 & 55,1 & 101,7 & 174,0 & 210,2 & 1,4 & 0,96 & 13,1 & 30,8 & 79,1 & 84,3 \\
\hline D3 & 3 & 0,7411 & 56,9 & 104,1 & 171,3 & 206,4 & 1,2 & 0,87 & 23,2 & 24,5 & 79,5 & 83,8 \\
\hline D4 & 4 & 0,7431 & 53,8 & 101,2 & 176,3 & 209,4 & 1,3 & 0,82 & 21,7 & 36,9 & 81,0 & 87,0 \\
\hline D5 & 5 & 0,7427 & 55,7 & 105,0 & 174,0 & 206,0 & 1,2 & 0,80 & 21,3 & 27,6 & 79,6 & 85,5 \\
\hline D6 & 6 & 0,7465 & 56,0 & 104,5 & 178,5 & 205,6 & 1,2 & 0,66 & 22,5 & 26,4 & 81,7 & 86,6 \\
\hline D7 & 7 & 0,7455 & 55,9 & 103,8 & 178,4 & 206,8 & 1,2 & 0,76 & 20,3 & 27,5 & 81,9 & 86,3 \\
\hline D8 & 8 & 0,7457 & 55,8 & 105,3 & 175,7 & 204,8 & 1,1 & 0,65 & 21,5 & 26,4 & 81,5 & 86,3 \\
\hline \multicolumn{13}{|l|}{ Abril } \\
\hline D1 & 9 & 0,7446 & 54,5 & 105,2 & 184,1 & 209,2 & 1,3 & 0,72 & 19,5 & 35,1 & 80,2 & 85,9 \\
\hline D2 & 10 & 0,7421 & 56,3 & 102,1 & 171,4 & 209,7 & 1,3 & 0,84 & 13,3 & 29,4 & 79,0 & 84,6 \\
\hline D3 & 11 & 0,7408 & 55,8 & 103,7 & 172,8 & 207,3 & 1,2 & 0,79 & 23,2 & 25,8 & 79,4 & 83,8 \\
\hline D4 & 12 & 0,7455 & 54,2 & 102,4 & 175,1 & 207,6 & 1,2 & 0,84 & 22,6 & 35,1 & 81,1 & 86,7 \\
\hline D5 & 13 & 0,7408 & 54,8 & 104,0 & 171,6 & 203,3 & 1,1 & 0,76 & 21,1 & 26,4 & 79,3 & 85,2 \\
\hline D6 & 14 & 0,7457 & 54,9 & 105,8 & 176,3 & 204,0 & 1,1 & 0,70 & 22,6 & 26,1 & 81,8 & 86,4 \\
\hline D7 & 15 & 0,7465 & 54,7 & 105,3 & 176,9 & 206,7 & 1,2 & 0,68 & 20,3 & 27,5 & 81,8 & 86,1 \\
\hline D8 & 16 & 0,7476 & 55,1 & 105,0 & 173,2 & 205,0 & 1,2 & 0,62 & 21,3 & 26,8 & 81,7 & 86,7 \\
\hline \multicolumn{13}{|l|}{ Maio } \\
\hline D1 & 17 & 0,7459 & 53,1 & 105,7 & 183,5 & 211,0 & 1,4 & 0,66 & 21,8 & 36,3 & 80,3 & 86,2 \\
\hline D2 & 18 & 0,7465 & 55,7 & 103,5 & 171,2 & 208,2 & 1,3 & 0,90 & 13,6 & 29,0 & 79,2 & 84,5 \\
\hline D3 & 19 & 0,7421 & 56,6 & 105,3 & 170,3 & 204,1 & 1,1 & 0,83 & 24,2 & 23,1 & 79,8 & 84,0 \\
\hline D4 & 20 & 0,7438 & 54,4 & 101,7 & 176,4 & 208,3 & 1,3 & 0,77 & 22,8 & 35,3 & 81,0 & 86,7 \\
\hline D5 & 21 & 0,7419 & 56,1 & 106,1 & 173,4 & 204,8 & 1,1 & 0,74 & 21,0 & 26,7 & 79,1 & 85,2 \\
\hline D6 & 22 & 0,7468 & 55,2 & 104,1 & 177,1 & 204,1 & 1,1 & 0,71 & 22,5 & 26,0 & 81,8 & 86,6 \\
\hline D7 & 23 & 0,7464 & 55,2 & 105,8 & 176,4 & 205,9 & 1,2 & 0,73 & 20,4 & 26,9 & 81,8 & 86,2 \\
\hline D8 & 24 & 0,7481 & 55,3 & 106,4 & 174,3 & 206,1 & 1,2 & 0,70 & 21,0 & 27,1 & 81,5 & 86,5 \\
\hline
\end{tabular}

